Please do not remove this page

RMIT

UNIVERSITY

\title{
Wide-band variable transversal phase-shifter
}

Bui, Lam; Mitchell, Arnan; Slade, Bill; Chio, T

https://researchrepository.rmit.edu.au/esploro/outputs/9921861587701341/filesAndLinks?institution=61RMIT_INST\&index=null

Bui, L., Mitchell, A., Slade, B., \& Chio, T. (2003). Wide-band variable transversal phase-shifter. International Microwave Symposium Digest.

https://researchrepository.rmit.edu.au/discovery/fulldisplay/alma9921861587701341/61RMIT_INST:Resea rchRepository

Repository homepage: https://researchrepository.rmit.edu.au

(c) 2003 IEEE. Personal use of this material is permitted. However, permission to reprint/republish this material for advertising or promotional purposes or for creating new collective works for resale or redistribution to servers or lists, or to reuse any copyrighted component of this work in other works must be obtained from the IEEE.

Downloaded On 2023/04/26 23:47:23 +1000 


\title{
Wide-band Variable Transversal Phase-shifter
}

\author{
Lam Anh Bui ${ }^{1}$, Arnan Mitchell ${ }^{1}$, G. William Slade ${ }^{1}$, and Tan-Huat Chio $^{2}$ \\ ${ }^{1}$ Australian Photonics CRC, RMIT University, GPO Box 2476V, Melbourne VIC 3001, Australia. \\ ${ }^{2}$ DSO National Laboratories, S(118230) Singapore.
}

\begin{abstract}
We present a novel broadband phase-shifter based on a transversal filter configuration. This approach allows flexible control of the amplitude response while providing continuous variation of a linear phase slope. Numerical examples, both ideal and using practical RF components are presented and practical challenges in realising the phase-shifter are identified.
\end{abstract}

\section{INTRODUCTION}

Many RF and microwave systems such as high resolution phased array radar [1], antenna remoting [2] and wide-band signal processing [3] require frequency linear or true time delay (TTD) phase-shifting. TTD implementations include switched delay lines (SDLs) [4], dispersive fiber prism [5] and fiber Bragg grating [6] approaches.

Of these, SDLs are the most conceptually simple and often most practical solutions to implement. SDLs are restricted to a finite number of predetermined phase shifts limiting beam steering capabilities [7]. Higher resolution SDLs have been devised, however, for arrays with a large number of elements and broad steering range, the number of distinct delay lines required could become cumbersome.

Recently we have proposed [8] and demonstrated [9] a variable feedback photonic phase-shifter (VFPPS). The VFPPS is a compact device exhibiting continuously variable, linear phase response. It can, however, exhibit serious amplitude imbalance [8].

In this paper, we propose a phase-shifting approach, which can provide the phase shifting performance of the VFPPS, while also maintaining good transmission over the range of operation. The proposed approach is based on a transversal filter configuration and is hence termed a transversal phase-shifter (TPS).

This paper is organised as follows. In Section II, the general TPS concept and principle of operation are presented. In Section III, a design methodology for the realization of practical phase shifters is presented. A phase-shifter operating from $0.2-2 \mathrm{GHz}$ is used as an illustrative example. Section IV presents simulations of a first attempt to realise the proposed phase shifter using

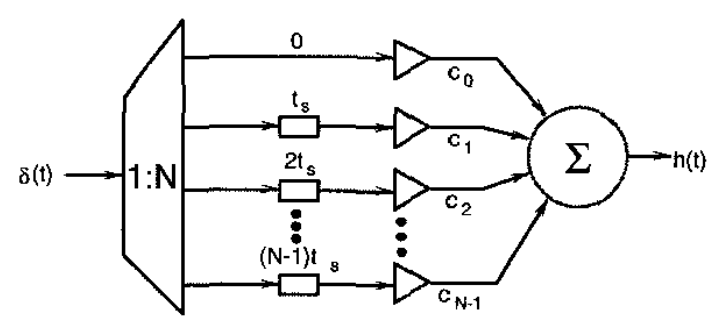

Fig.1 Schematic of a generic $\mathbf{N}$ tap transversal phase-

practical electronic components. A discussion of the findings of this paper and directions for further work are presented in Section V.

\section{TPS THEORY}

The proposed phase shifter has the same form as the well known transversal filters [10]. The input power is split among $\mathrm{N}$ taps which each contribute a different time delay. The $\mathrm{N}$ delayed components are then summed at the output. By controlling the signal amplitude in each tap, it is possible to engineer an arbitrary filter response. A schematic of the TPS is presented in Fig.1.

We propose that such a system could be used as a phase shifter as shown in Fig.2. Initially the tap coefficients are arranged as depicted in Fig.2a) to provide a transfer function with a desired frequency response. The tap coefficients can be considered the impulse response of the desired frequency response.

A phase shift in the frequency response is equivalent to a time shift of the impulse response. This can be achieved by using the same delay lines with different coefficients to approximate a time shifted version of the original impulse response as shown in Fig.2b).

As the impulse response is translated from its symmetric central position, the phase linearity of the frequency response will degrade. This will be particularly acute as the impulse response exceeds the range of available taps. Further, since there is only a finite number of taps, it will be important to represent the impulse 


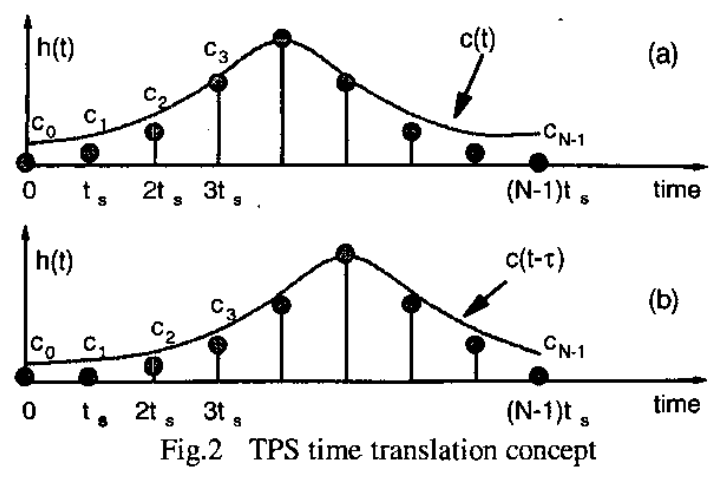

response compactly. Thus windowing of the impulse response will play an important role in the realisation of broadly variable . devices with tolerable phase and amplitude response fluctuation.

\section{TRansversal Phase ShIFTer Design}

In order to examine the design of a TPS device and to explore the range over which such a device may be practically implemented, a specific example phase shifter will be developed. This example is based on a published investigation in which a switched delay line phase shifter was employed to provide beam steering for radio astronomy applications in the frequency range $0.2-2 \mathrm{GHz}$ $[7,11]$. It was found [7] that this phasing system was limited in the number of distinct beam angles that could be produced. It is predicted that the TPS approach should be able to provide continuous phase shifting which will address this issue and hence this seems a worthy example.

The criteria chosen for this example were - frequency range: $0.2-2 \mathrm{GHz}$; phase shift range: $360^{\circ}$ at $2 \mathrm{GHz}$ (linear with frequency); phase error $<6^{\circ}$; magnitude ripple $<1 \mathrm{~dB}$ over the frequency range. A TPS that meets these specifications was designed as follows.

\section{A. Establish basic filter response}

The design begins by identifying the desired frequency response for the TPS. Due to the very broad bandwidth required $(0.2-2 \mathrm{GHz})$ a low pass filter prototype is chosen, and hence the resulting TPS will operate from DC up to $2 \mathrm{GHz}$. The precise cut off frequency of the filter may be made slightly greater than $2 \mathrm{GHz}$ in order to optimise the TPS as will be discussed later.

Ideally, we desire a magnitude response with minimal ripple from $\mathrm{DC}-2 \mathrm{GHz}$, but are not concerned with the behaviour outside this range. It is, however, important that the impulse response is compactly defined such that the number of taps required is as small as possible.
The Kaiser window has been specifically developed for this purpose [12]. It is parameterized by a single quantity $\alpha$. Algorithms are available to relate this parameter to the maximum passband ripple, the minimum stop band attenuation, and the transition bandwidth of the desired response [12]. As an initial condition for our example we have set passband ripple $<1 \mathrm{~dB}$, stop band attenuation $>10 \mathrm{~dB}$ and transition bandwidth of $10 \%$.

\section{B. Establish delay line increment}

Algorithms to determine the sampling frequency and number of taps for filters employing Kaiser windows are also available [12]. Using the initial conditions identified above, it was found that the time delay increment required for our example was $t_{s}=180$ ps and thus 5 taps are required to adequately represent the desired frequency response.

\section{Establish required number of delay lines}

It is worth noting that the identified impulse response is to be time shifted to achieve phase shifting as depicted in Fig.2b). Hence extra taps will be required to sustain the frequency characteristics over the full range of phase shifts. The range of time shifts required can be calculated from the specified phase range as $\tau_{\max }=\theta_{\max } /\left(2 \pi \mathrm{f}_{\max }\right)$, thus $\tau_{\max }=500$ ps. Using the sampling period identified above, it is evident that the approximate total number of taps should be 7 .

It is also anticipated that intermediate time shifting of the impulse response will introduce asymmetry. Thus it may also be necessary to increase the sampling frequency such that any excess ripple will remain within tolerable bounds.

\section{Empirical optimisation of TPS parameters}

Having identified the approximate number of taps required to represent the desired frequency response over the specified range of phase shift, it is now necessary to optimise the parameters to ensure the design specifications can be met with a minimum number of taps. Here the optimisation is performed empirically; however it is expected that implementation of a numerical optimisation algorithm for this purpose should pose no great challenge.

The optimisation variables are the cut off frequency of the low pass filter prototype, the Kaiser window parameter $\alpha$, the total number of delay lines, and the delay line length increment. The optimization goal is that all of the design specifications are met continuously across the range of phase shifting. To ensure that the specified performance is maintained for all phasing 


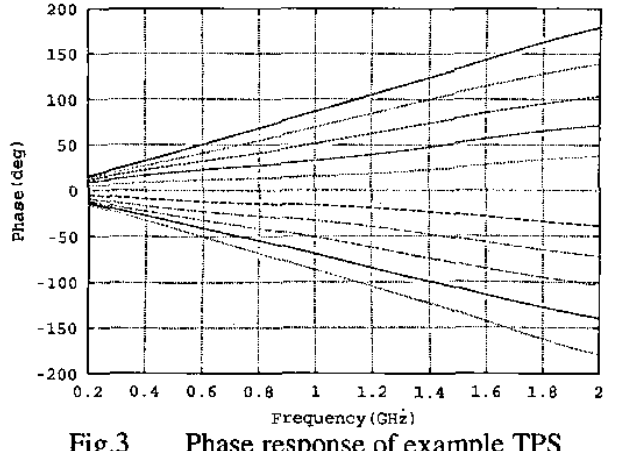

Fig. 3 Phase response of example TPS

values, the range of time shifts is divided into eleven equal segments. Both the phase and frequency response of the TPS is then calculated using a simple computer algorithm for each of these settings. Holding the number of taps constant, the three remaining variables are adjusted until the design specifications are met for all settings. The number of taps can then be reduced and the optimisation repeated.

The phase response of the optimal solution with 7 taps is presented in Fig.3. The phase response is quite linear over the frequency range with a deviation of less than $5^{\circ}$ maintained over the band. The magnitude response for the same phase shifts is also presented in Fig.4. The ripple remains within $1 \mathrm{~dB}$ for the entire frequency band indicating that the design criteria have been met for 7 taps.

\section{NUMERICAL DEMONSTRATION OF AN ELECTRONTC IMPLEMENTATION}

In order to illustrate the behaviour of a practically realizable TPS it is desired to model the system shown in Fig.1 using transmission line segments as well as ideal and non-ideal multipliers (for generating the coefficients $c_{n}$ ). The use of ideal multipliers in the simulations validate the convergence of the numerical model and set the baseline performance of the TPS for a given set of chosen coefficients. It is expected that the non-ideal multipliers (which are modeled here as balanced diode multipliers) will exhibit significant distortion of the input signal, but this should not detract from the phase-shifting behaviour of the TPS. Future work will involve implementing wide-band dispersion compensation as well as linearization techniques on the multipliers. Some of this work will be addressed in the presentation.

Given the proper choice of coefficients, delay times, which are not integer multiples of the "sample times", are exhibited. Table I lists 11 sets of coefficients, which produce time shifts in steps of $48.6 \mathrm{ps}$.

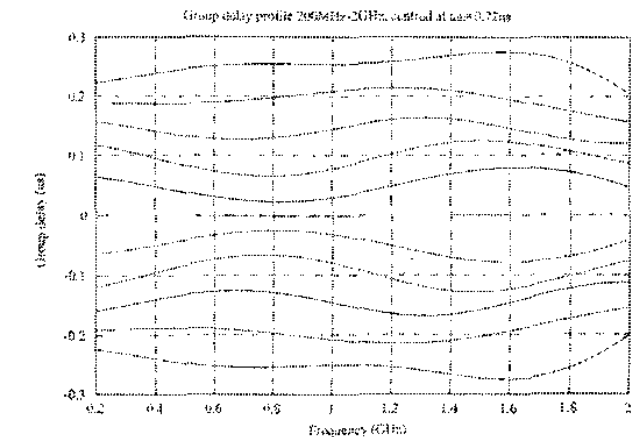

Fig.5 Group delay distribution for the example TPS with ideal multipliers. Each curve corresponds to a row in

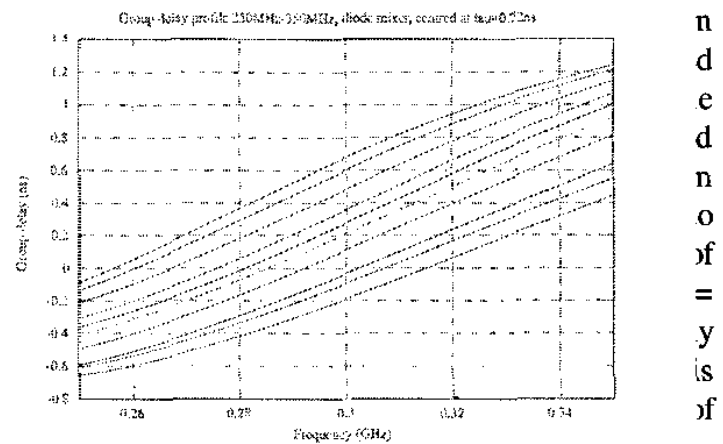

Fig.6 Group delay distribution over a 100MHz band for the example TPS using non-ideal multipliers (diode balanced mixers) to generate the coefficients

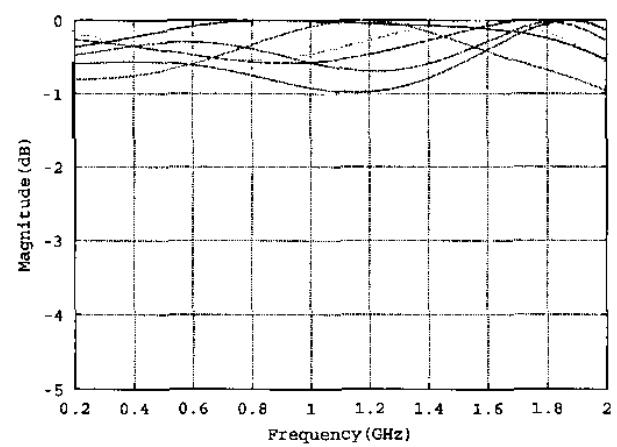

Fig.4 Normalised Magnitude Response of example 
TABLE I

LIST OF COEFFICIENTS FOR GENERATING TIME DELAYS. THE FIRST COLUMN REPRESENTS THE DEVIATION FROM THE DELAY TIME OF THE CENTRAL TAP IN FIG. $1(\tau=0.72 \mathrm{~ns})$. NOTE THAT THESE DELAY TIMES ARE NOT INTEGER MULTIPLES OF THE TAP DELAYS.

\begin{tabular}{|c|c|c|c|c|c|c|c|}
\hline$\Delta \mathrm{t}(\mathrm{ns})$ & $\mathrm{c}_{0}$ & $\mathrm{c}_{1}$ & $\mathrm{c}_{2}$ & $\mathrm{c}_{3}$ & $\mathrm{c}_{4}$ & $\mathrm{c}_{5}$ & $\mathrm{c}_{6}$ \\
\hline-0.243 & -0.126936 & 0.385979 & 0.760821 & -0.188998 & 0.093797 & -0.050724 & 0.026193 \\
\hline-0.194 & -0.053439 & 0.105566 & 0.913985 & -0.033998 & -0.001479 & 0.011892 & -0.013741 \\
\hline-0.146 & 0.031323 & -0.09841 & 0.873228 & 0.252768 & -0.124114 & 0.078365 & -0.050106 \\
\hline-0.0972 & 0.079987 & -0.176631 & 0.65408 & 0.588478 & -0.198252 & 0.104591 & -0.058191 \\
\hline-0.0486 & 0.073816 & -0.136936 & 0.338516 & 0.857369 & -0.153123 & 0.063648 & -0.028098 \\
\hline 0 & 0.026622 & -0.033662 & 0.038354 & 0.96 & 0.038354 & -0.033662 & 0.026622 \\
\hline 0.0486 & -0.028098 & 0.063648 & -0.153123 & 0.857369 & 0.338516 & -0.136936 & 0.073816 \\
\hline 0.0972 & -0.058191 & 0.104591 & -0.198251 & 0.588478 & 0.65408 & -0.176631 & 0.079987 \\
\hline 0.146 & -0.050106 & 0.078365 & -0.124114 & 0.252768 & 0.873228 & -0.09841 & 0.031323 \\
\hline 0.194 & -0.013741 & 0.011892 & -0.001479 & -0.033998 & 0.913985 & 0.105566 & -0.053439 \\
\hline 0.243 & 0.026193 & -0.050724 & 0.093797 & -0.188998 & 0.760821 & 0.385279 & -0.126936 \\
\hline
\end{tabular}

coefficients). Fig. 6 shows the delay for the same system topology, but a diode balanced mixer is used in place of the ideal multiplier. Because the non-ideal mixer is strongly dispersive over the $0.2-2 \mathrm{GHz}$ band, a narrower band is depicted in Fig.6.The stepped delay times are clearly evident, thereby demonstrating the possibility of realising TPSs using readily available components. However, strategies for mitigating dispersive effects of electronic components need to be devised if wide-band TPSs are to be constructed. One obvious method would be the use of a photonic system [13].

\section{CONCLUSIONS}

A novel broadband phase-shifter based on a transversal filter structure has been proposed. The phase-shifter exhibits minimal amplitude imbalance and frequency linear phase-shift. A phase shifter with only 7 taps was designed for $0.2-2 \mathrm{GHz}$ frequency range. Modeling of this device with transmission lines and balanced diode mixers demonstrated that time shifting could be achieved practically, however, the strong dispersion of the diodes mixers limited the device bandwidth. It is therefore suggested that techniques to linearise/compensate dispersion of the multipliers should be considered. Alternatively, a photonic realisation of the TPS should also be attempted.

\section{REFERENCES}

[1] A. N. Riza and J. B. Thompson, "Selected papers on Photonic Control Systems for Phased Array Antennas," SPIE Press, 1997.

[2] P. J. Matthews and P. D. Biernacki, "Photonic signal processing for microwave applications," 1999 IEEE MTT-S Int. Microwave Symp. Dig., pp. 877-880 Vol. 3, 1999.
[3] B. Moslehi, J. W. Goodman, M. Tur, and H. J. Shaw, "Fiber-optic lattice signal processing," Proceedings of the IEEE, vol. 72, pp. 909-930, 1984.

[4] A. Ouacha, B. Carlegrim, and M. Alfredson, "Ultra broadband MMIC 5-bit time shifter," 1999 Asia Pacific Microwave Conference., pp. 242-245 Vol. 2, 1999.

[5] R. D. Esman, M. Y. Frankel, J. L. Dexter, L. Goldberg, M. G. Parent, D. Stilwell, and D. G. Cooper, "Fiber-optic prism true time-delay antenna feed," IEEE Photonics Technology Letters, vol. 5, pp. 1347-1349, 1993.

[6] J. L. Corral, J. Marti, S. Regidor, J. M. Foster, R. Laming, and M. J. Cole, "Continuously variable true time-delay optical feeder for phased-array antenna employing chirped fiber grating," IEEE Trans. Microwave Theory and Tech., vol. 45 , pp. 1531-1536, 1997.

[7] G. A. Hampson, A. B. Smolders, and G. W. Kant, "Hierarchical beamforming aspects of OSMA," ISSPA '99. Proceedings of the Fifth Int. Symp.. on Signal Processing and its Applications, pp. 869-872 Vol. 2, 1999.

[8] K. Ghorbani, A. Mitchell, R. B. Waterhouse, and M. W. Austin, "A novel wide-band tunable RF phase shifter using a variable optical directional coupler," IEEE Trans. on Microwave Theory and Tech., vol. 47, pp. 645-648, 1999.

[9] A. Mitchell, K. Ghorbani, M. Austin, and R. Waterhouse, "Integrated optic RF phase shifter for continuous beam steering at 1-1200 MHz," 2000 IEEE MTT-S Int. Microwave Symp. Dig., pp. 699-702 Vol. 2, 2000.

[10] E. H. Kallmann, "Transversal Filters," Proceedings of the I.R.E., pp. 302-310, 1940.

[11] B. Smolders and G. Hampson, "Deterministic RF nulling in phased arrays for the next generation of radio telescopes," - IEEE Antennas and Propagation Magazine, vol. 44, pp. 13-22, 2002.

[12] J. F. Kaiser, "Nonrecursive digital filter design using the 10-sinh window function," Proceedings of the 1974 IEEE Int. Symp. on Circuits and Systems, pp. 20-23, 1974.

[13] K. P. Jackson, S. A. Newton, B. Moslehi, M. Tur, C. C. Cutler, J. W. Goodman, and H. J. Shaw, "Optical fiber delay-line signal processing," IEEE Trans Microwave Theory and Tech., vol. MTT-33, pp. 193-210, 1985. 